\title{
Three Different Methods for New Soliton Solutions of the Generalized NLS Equation
}

\author{
Anwar Ja'afar Mohamad Jawad \\ Al-Rafidain University College, Baghdad 00964, Iraq \\ Correspondence should be addressed to Anwar Ja’afar Mohamad Jawad; anwar_jawad2001@yahoo.com
}

Received 23 June 2017; Revised 2 September 2017; Accepted 20 September 2017; Published 18 October 2017

Academic Editor: Jozef Banas

Copyright (c) 2017 Anwar Ja'afar Mohamad Jawad. This is an open access article distributed under the Creative Commons Attribution License, which permits unrestricted use, distribution, and reproduction in any medium, provided the original work is properly cited.

\begin{abstract}
Three different methods are applied to construct new types of solutions of nonlinear evolution equations. First, the Csch method is used to carry out the solutions; then the Extended Tanh-Coth method and the modified simple equation method are used to obtain the soliton solutions. The effectiveness of these methods is demonstrated by applications to the RKL model, the generalized derivative NLS equation. The solitary wave solutions and trigonometric function solutions are obtained. The obtained solutions are very useful in the nonlinear pulse propagation through optical fibers.
\end{abstract}

\section{Introduction}

Partial differential equations describe various nonlinear phenomena in natural and applied sciences such as fluid dynamics, plasma physics, solid state physics, optical fibers, acoustics, biology, and mathematical finance. Partial differential equations which arise in real-world physical problems are often too complicated to be solved exactly. It is of significant importance to solve nonlinear partial differential equations (NLPDEs) from both theoretical and practical points of view. The analysis of some physical phenomena is investigated by the exact solutions of nonlinear evolution equations (NLEEs) [1-9].

In this paper, the third-order generalized NLS equation is studied, which is proposed by Radhakrishnan, Kundu, and Lakshmanan (RKL) [10]. The normalized RKL model can be written as

$$
\begin{aligned}
i q_{t}+ & q_{x x}+2|q|^{2} q+i \alpha q_{x x x}+i \beta\left(|q|^{2} q\right)_{x} \\
& +i \gamma\left(|q|^{4} q\right)_{x}+\delta|q|^{4} q=0 .
\end{aligned}
$$

Equation (1) describes the propagation of femtosecond optical pulses, $q(x, t)$ represents normalized complex slowly varying amplitude of the pulse envelope, and $\alpha, \beta, \gamma$, and $\delta$ are real constants. Some solitary wave solutions and combined Jacobian elliptic function solution were constructed by different methods $[3,4]$.

The Csch method is used to carry out the solutions. Then, the Extended Tanh-Coth method and the modified simple equation method are used to obtain the soliton solutions of this equation.

\section{Traveling Wave Solution}

Consider the nonlinear partial differential equation in the form

$$
F\left(u, u_{t}, u_{x}, u_{t t}, u_{x x}, u_{x x x}, \ldots\right)=0
$$

where $u(x, t)$ is a traveling wave solution of nonlinear partial differential equation (2). We use the transformations,

$$
u(x, t)=f(\xi),
$$

where $\xi=k x-\lambda t$. This enables us to use the following changes: 


$$
\begin{aligned}
& \frac{\partial}{\partial t}(\cdot)=-\lambda \frac{d}{d \xi}(\cdot), \\
& \frac{\partial}{\partial x}(\cdot)=k \frac{d}{d \xi}(\cdot) .
\end{aligned}
$$

Using (4) to transfer the nonlinear partial differential equation (2) to nonlinear ordinary differential equation,

$$
Q\left(f, f^{\prime}, f^{\prime \prime}, f^{\prime \prime \prime}, \ldots\right)=0 .
$$

The ordinary differential equation (5) is then integrated as long as all terms contain derivatives, where we neglect the integration constants.

\section{The Generalized NLS Equation (RKL)}

In this section, the generalized third-order NLS equation (RKL) (1) is chosen to illustrate the effectiveness of three methods.

The solution of (1) may be supposed as

$$
q(x, t)=e^{i \theta} u(\xi)
$$

where $\theta=k_{1} x+k_{2} t, \xi=k x-\lambda t$.

Substituting (6) into (1) and by defining the derivatives,

$$
\begin{aligned}
& q_{t}=\left[-\lambda u^{\prime}(\xi)+i k_{2} u(\xi)\right] e^{i \theta}, \\
& q_{x}=\left[k u^{\prime}(\xi)+i k_{1} u(\xi)\right] e^{i \theta}, \\
& q_{x x}=\left[k^{2} u^{\prime \prime}+2 i k k_{1} u^{\prime}-k_{1}^{2} u\right] e^{i \theta}, \\
& q_{x x x}=\left[k^{3} u^{\prime \prime \prime}+2 i k^{2} k_{1} u^{\prime \prime}-k k_{1}{ }^{2} u^{\prime}+i k_{1} k^{2} u^{\prime \prime}\right. \\
& \left.\quad-2 k k_{1}{ }^{2} u^{\prime}-i k_{1}^{3} u\right] e^{i \theta} ;
\end{aligned}
$$

$$
\begin{aligned}
& \delta=\left(\frac{1}{\alpha}+2 k_{1}\right) \gamma, \\
& \beta=2 \alpha,
\end{aligned}
$$$$
\alpha=\frac{-\left[k k_{2}-8 k k_{1}{ }^{2}+3 \lambda k_{1}\right] \mp \sqrt{\left[k k_{2}-8 k k_{1}^{2}+3 \lambda k_{1}\right]^{2}+32 k k_{1}^{3}\left(\lambda-2 k k_{1}\right)}}{16 k k_{1}^{3}}
$$

then decomposing (1) into real and imaginary parts yields a pair of relations which represented nonlinear ordinary differential equations. The real part is

$$
\begin{aligned}
k^{2}[1 & \left.-3 \alpha k_{1}\right] u^{\prime \prime}+\left[\alpha k_{1}{ }^{3}-k_{1}{ }^{2}-k_{2}\right] u \\
& +\left[2-3 k_{1} \beta\right] u^{3}+\left[\delta-5 k_{1} \gamma\right] u^{5}=0
\end{aligned}
$$

while the imaginary part is

$$
\begin{aligned}
& \alpha k^{3} u^{\prime \prime \prime}+\left[2 k k_{1}-\lambda-3 \alpha k k_{1}^{2}\right] u^{\prime}+3 \beta k u^{2} u^{\prime} \\
& +5 k \gamma u^{4} u^{\prime}=0 .
\end{aligned}
$$

Integrating (9) once and setting the integration constant to zero, we obtain

$$
\alpha k^{3} u^{\prime \prime}+\left[2 k k_{1}-\lambda-3 \alpha k k_{1}^{2}\right] u+\beta k u^{3}+k \gamma u^{5}=0 .
$$

Equations (8) and (10) will be equivalent, provided that

$$
\begin{aligned}
\frac{k^{2}\left[1-3 \alpha k_{1}\right]}{\alpha k^{3}} & =\frac{\left[\alpha k_{1}^{3}-k_{1}{ }^{2}-k_{2}\right]}{\left[2 k k_{1}-\lambda-3 \alpha k k_{1}^{2}\right]}=\frac{\left[2-3 \beta k_{1}\right]}{\beta k} \\
& =\frac{\left[\delta-5 \gamma k_{1}\right]}{k \gamma},
\end{aligned}
$$

from which we get the parametric constraints multiplying both sides of (10) by $u^{\prime}$ and integrating with respect to $\xi$ with zero constant, we get

$$
\begin{aligned}
& u^{\prime 2}+\frac{\beta\left[\alpha k_{1}{ }^{3}-k_{1}{ }^{2}-k_{2}\right]}{\alpha k^{2}} u^{2}+\frac{\beta}{2 \alpha k^{2}} u^{4}+\frac{\gamma}{3 \alpha k^{2}} u^{6} \\
& \quad=0
\end{aligned}
$$

assume that

$$
\begin{aligned}
& c_{2}=\frac{\beta\left[\alpha k_{1}^{3}-k_{1}^{2}-k_{2}\right]}{\alpha k^{2}}, \\
& c_{4}=\frac{\beta}{2 \alpha k^{2}}, \\
& c_{6}=\frac{\gamma}{3 \alpha k^{2}} .
\end{aligned}
$$


Then

$$
u^{\prime 2}+c_{2} u^{2}+c_{4} u^{4}+c_{6} u^{6}=0
$$

\section{Methodology}

In this section we will apply three different methods to solve (15). These methods are Csch method, Extended Tanh-Coth method, and the modified simple equation method (MSEM).

4.1. Csch Function Method. The solution of many nonlinear equations can be expressed in the form [11]

$$
u(\xi)=A \operatorname{csch}^{\tau}(\mu \xi)
$$

and their derivative

$$
\begin{aligned}
u^{\prime}(\xi) & =-A \tau \mu \operatorname{csch}^{\tau}(\mu \xi) \cdot \operatorname{coth}(\mu \xi), \\
u^{\prime \prime}(\xi) & =A \tau \mu^{2}\left[(\tau+1) \operatorname{csch}^{\tau+2}(\mu \xi)+\tau \operatorname{csch}^{\tau}(\mu \xi)\right],
\end{aligned}
$$

where $A, \mu$, and $\tau$ are parameters to be determined and $\mu$ and $\lambda$ are the wave number and the wave speed, respectively. We substitute (16)-(17) into the reduced equation (15); we get

$$
\begin{aligned}
& A^{2} \tau^{2} \mu^{2} \operatorname{csch}^{2 \tau}(\mu \xi)+A^{2} \tau^{2} \mu^{2} \operatorname{csch}^{2 \tau+2}(\mu \xi) \\
& +c_{2} A^{2} \operatorname{csch}^{2 \tau}(\mu \xi)+c_{4} A^{4} \operatorname{csch}^{4 \tau}(\mu \xi) \\
& +c_{6} A^{6} \operatorname{csch}^{6 \tau}(\mu \xi)=0 .
\end{aligned}
$$

$$
q(x, t)=\mp \sqrt[4]{\frac{3 \beta}{\gamma}\left[\alpha k_{1}^{3}-k_{1}{ }^{2}-k_{2}\right]} e^{i\left[k_{1} x+k_{2} t\right]} \sqrt{\operatorname{csch}\left(\frac{2 i}{k} \sqrt{\frac{\beta}{\alpha}\left[\alpha k_{1}{ }^{3}-k_{1}{ }^{2}-k_{2}\right]}(k x-\lambda t)\right)} ;
$$

Figure 1 represents the solitary wave in (23) for $k=k_{1}=k_{2}=$ $1, \lambda=-1, \gamma=-1, \delta=-10 / 3, \beta=3 / 2, \alpha=3 / 4$, and then $q(x, t)=1.54 \sqrt{\operatorname{csch}(3.1622(x+t))}$.

4.2. Tanh-Coth Method. The key step is to introduce the ansatz, the new independent variable $[12,13]$

$$
Y=\tanh (\xi)
$$

that leads to the change of variables:

$$
\begin{aligned}
\frac{d U}{d \xi}= & \left(1-Y^{2}\right) \frac{d U}{d Y}, \\
\frac{d^{2} U}{d \xi^{2}}= & -2 Y\left(1-Y^{2}\right) \frac{d U}{d Y}+\left(1-Y^{2}\right)^{2} \frac{d^{2} U}{d Y^{2}}, \\
\frac{d^{3} U}{d \xi^{3}}= & 2\left(1-Y^{2}\right)\left(3 Y^{2}-1\right) \frac{d U}{d Y} \\
& -6 Y\left(1-Y^{2}\right)^{2} \frac{d^{2} U}{d Y^{2}}+\left(1-Y^{2}\right)^{3} \frac{d^{3} U}{d Y^{3}} .
\end{aligned}
$$

Balance the terms of the Csch functions to find $\tau$

$$
2 \tau+2=6 \tau, \quad \text { Then } \tau=\frac{1}{2} .
$$

We next collect all terms in (18) with the same power in $\operatorname{csch}^{k}(\mu \xi)$ and set their coefficients to zero to get a system of algebraic equations among the unknowns $A, \mu$, and $\tau$ and solve the subsequent system

$$
\begin{aligned}
& A^{2} \frac{1}{4} \mu^{2}+c_{2} A^{2}=0, \\
& A^{2} \frac{1}{4} \mu^{2}+c_{6} A^{6}=0 .
\end{aligned}
$$

Solving the system of equations in (20), we get

$$
\begin{aligned}
& \mu=2 i \sqrt{c_{2}}=\frac{2 i}{k} \sqrt{\frac{\beta}{\alpha}\left[\alpha k_{1}^{3}-k_{1}{ }^{2}-k_{2}\right]}, \\
& A=\mp \sqrt[4]{\frac{c_{2}}{c_{6}}}=\mp \sqrt[4]{\frac{3 \beta}{\gamma}\left[\alpha k_{1}^{3}-k_{1}{ }^{2}-k_{2}\right]} ;
\end{aligned}
$$

then

$$
u(\xi)=\mp \sqrt[4]{\frac{3 \beta}{\gamma}\left[\alpha k_{1}^{3}-k_{1}^{2}-k_{2}\right]} \sqrt{\operatorname{csch}(\mu \xi)}
$$

therefore
Assume

$$
V(\xi)=u^{2}(\xi) .
$$

Equation (15) can be written as

$$
V^{\prime 2}+4 c_{2} V^{2}+4 c_{4} V^{3}+4 c_{6} V^{4}=0 .
$$

The next step is that the solution of (29) is expressed in the form

$$
V(\xi)=\sum_{i=0}^{m} a_{i} Y^{i}+\sum_{i=1}^{m} b_{i} Y^{-i},
$$

where the parameter $m$ can be found by balancing the highest-order linear term with the nonlinear terms in (29).

We balance $V^{4}$ with $(d V / d Y)^{2}$, to obtain $4 m=(m+1)^{2}$; then $m=1$. The Tanh-Coth method admits the use of the finite expansion for

$$
\begin{aligned}
V & =a_{0}+a_{1} Y+b_{1} Y^{-1}, \\
V^{\prime} & =a_{1}-b_{1} Y^{-2} .
\end{aligned}
$$




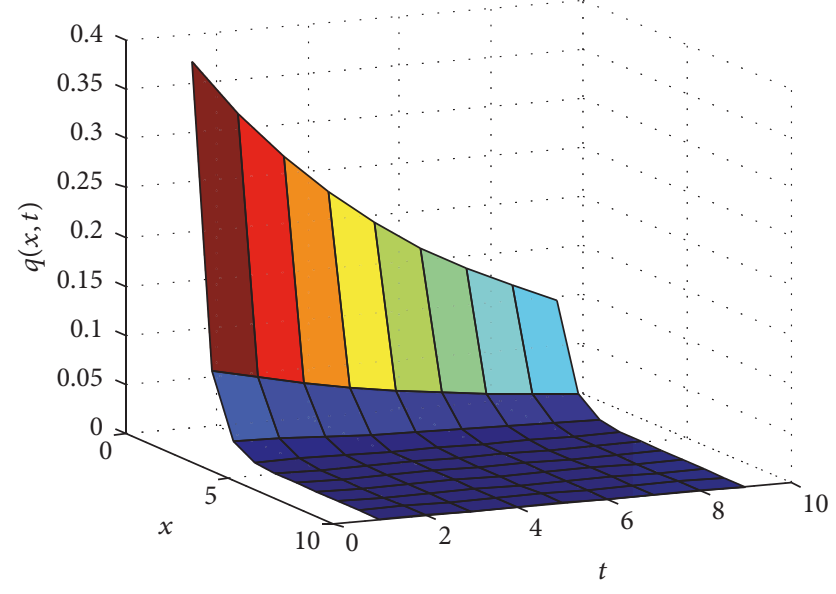

FIGURE 1: The solitary wave in (23) for $0 \leq t \leq 1,0 \leq x \leq 10$.

$\lambda, k, a_{0}, a_{1}, b_{1}$ are to be determined. Substituting (31) into (25) and then into (29) will yield a set of algebraic equations because all coefficients of $Y$ have to vanish

$$
\begin{aligned}
& \left(1-Y^{2}\right)^{2}\left(a_{1}-b_{1} Y^{-2}\right)^{2}+4 c_{2}\left(\left[a_{0}+a_{1} Y\right]+b_{1} Y^{-1}\right)^{2} \\
& \quad+4 c_{4}\left(a_{0}+a_{1} Y+b_{1} Y^{-1}\right)^{3} \\
& \quad+4 c_{6}\left(a_{0}+a_{1} Y+b_{1} Y^{-1}\right)^{4}=0 .
\end{aligned}
$$

Equation (32) can be written as

$$
\begin{aligned}
& \left(a_{1}^{2}\left(1-2 Y^{2}+Y^{4}\right)-2 a_{1} b_{1}\left(Y^{-2}-2+Y^{2}\right)\right. \\
& \left.\quad+b_{1}^{2}\left(Y^{-4}-2 Y^{-2}+1\right)\right)+4 c_{2}\left(a_{0}^{2}+2 a_{1} b_{1}\right. \\
& \left.+2 a_{0} a_{1} Y+a_{1}^{2} Y^{2}+2 a_{0} b_{1} Y^{-1}+b_{1}^{2} Y^{-2}\right)+4 c_{4}\left(a_{0}^{3}\right. \\
& +3 a_{0}^{2} a_{1} Y+3 a_{0} a_{1}^{2} Y^{2}+a_{1}^{3} Y^{3}+3\left(a_{0}^{2} Y^{-1}\right. \\
& \left.+2 a_{0} a_{1}+a_{1}^{2} Y\right) b_{1}+3\left(a_{0} Y^{-2}+a_{1} Y^{-1}\right) b_{1}^{2} \\
& \left.+b_{1}{ }^{3} Y^{-3}\right)+4 c_{6}\left(\left[a_{0}^{4}+4 a_{0}^{3} a_{1} Y+6 a_{0}^{2} a_{1}^{2} Y^{2}\right.\right. \\
& \left.+4 a_{0} a_{1}^{3} Y^{3}+a_{1}^{4} Y^{4}\right]+4\left[a_{0}^{3} Y^{-1}+3 a_{0}^{2} a_{1}\right. \\
& \left.+3 a_{0} a_{1}^{2} Y+a_{1}^{3} Y^{2}\right] b_{1}+6\left[a_{0}^{2} Y^{-2}+2 a_{0} a_{1} Y^{-1}\right. \\
& \left.\left.\quad+a_{1}^{2}\right] b_{1}^{2}+4\left[a_{0} Y^{-3}+a_{1} Y^{-2}\right] b_{1}^{3}+b_{1}^{4} Y^{-4}\right)=0 .
\end{aligned}
$$

Equating expressions at $Y^{i},(i=-4,-3,-2,-1,0,1,2,3,4)$ to zero, we have the following system of equations:

Coefficients of $Y^{-4}:\left[1+4 c_{6} b_{1}{ }^{2}\right] b_{1}{ }^{2}=0$

Coefficients of $Y^{-3}:\left\{c_{4}+4 c_{6} a_{0}\right\} b_{1}^{3}=0$
Coefficients of $Y^{-2}:-2 a_{1} b_{1}-2 b_{1}{ }^{2}+4 c_{2} b_{1}{ }^{2}$

$$
+12 c_{4} a_{0} b_{1}^{2}+4 c_{6}\left(6 a_{0}^{2}+4 a_{1} b_{1}\right) b_{1}^{2}=0
$$

Coefficients of $Y^{-1}\left\{8 c_{2} a_{0}+12 c_{4}\left(a_{0}^{2}+a_{1} b_{1}\right)\right.$

$$
\left.+4 c_{6}\left(4 a_{0}^{3}+12 a_{0} a_{1} b_{1}\right)\right\} b_{1}=0
$$

Coefficients of $Y^{0}: a_{1}{ }^{2}+4 a_{1} b_{1}+b_{1}{ }^{2}+4 c_{2} a_{0}{ }^{2}$

$$
\begin{aligned}
& +4 c_{4}\left(a_{0}^{3}+6 a_{0} a_{1} b_{1}\right)+4 c_{6}\left(a_{0}^{4}+12 a_{0}{ }^{2} a_{1} b_{1}\right. \\
& \left.+6 a_{1}^{2} b_{1}^{2}\right)=0
\end{aligned}
$$

Coefficients of $Y: 8 c_{2} a_{0} a_{1}+12 c_{4}\left(a_{0}{ }^{2}+a_{1} b_{1}\right) a_{1}$

$$
+16 c_{6} a_{0} a_{1}\left(a_{0}^{2}+3 a_{1} b_{1}\right)=0
$$

Coefficients of $Y^{2}:-2 a_{1}^{2}-2 a_{1} b_{1}+4 c_{2} a_{1}{ }^{2}$

$$
+12 c_{4} a_{0} a_{1}^{2}+4 c_{6} a_{1}^{2}\left(6 a_{0}^{2}+4 a_{1} b_{1}\right)=0
$$

Coefficients of $Y^{3}:\left(c_{4}+4 c_{6} a_{0}\right) a_{1}^{3}=0$

Coefficients of $Y^{4}:\left(1+4 c_{6} a_{1}^{2}\right) a_{1}^{2}=0$.

Solving the system of equations (34), we get

$$
\begin{aligned}
& b_{1}=\mp \frac{i}{2 \sqrt{c_{6}}}, \\
& a_{0}=-\frac{1}{2 \sqrt{c_{6}}}, \\
& a_{1}=0, \\
& c_{2}=1, \\
& c_{4}=2 \sqrt{c_{6}} .
\end{aligned}
$$

Substitute for $c_{6}$ from (14), and then

$$
\begin{aligned}
& b_{1}=\mp i k^{2}, \\
& a_{0}=-k^{2}, \\
& a_{1}=0, \\
& c_{4}=\frac{1}{k} \sqrt{\frac{1}{k},} \\
& k=\sqrt{2\left[\alpha k_{1}^{3}-k_{1}^{2}-k_{2}\right]}, \\
& \gamma=\frac{3 \alpha}{4 k^{2}}, \\
& \delta=\frac{3\left(1+2 \alpha k_{1}\right)}{4 k^{2}} ;
\end{aligned}
$$




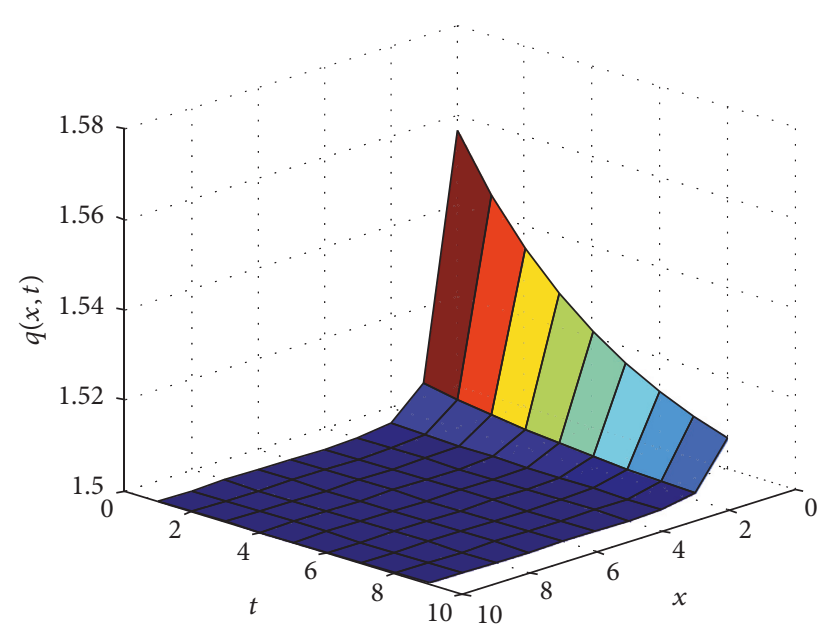

Figure 2: The solitary wave in (39) for $0 \leq t \leq 1,0 \leq x \leq 10$.

therefore

$$
\begin{gathered}
V(\xi)=k^{2}[-1 \mp i \operatorname{coth}(\xi)] \\
u(\xi)=k \sqrt{-1 \mp i \operatorname{coth}(\xi) .}
\end{gathered}
$$

Then

$$
q(x, t)=e^{i\left[k_{1} x+k_{2} t\right]} k \sqrt{-1 \mp i \operatorname{coth}(k x-\lambda t)}
$$

for $k=\sqrt{1 / 2}, k_{1}=k_{2}=1, \lambda=3 k, \alpha=1 / 4$, and then

$$
q(x, t)=\left(\frac{1}{\sqrt{2}}\right) \sqrt{-1+i \operatorname{coth}\left\{\left(\frac{1}{\sqrt{2}}\right)(x-3 t)\right\}} .
$$

Figure 2 represents the solitary wave in (39).

4.3. The Modified Simple Equation Method. We look for solutions of (29) in the form [14]

$$
\begin{aligned}
& V=A_{0}+A_{1} \frac{\psi_{\xi}}{\psi}, \\
& V_{\xi}=A_{1}\left(\frac{\psi_{\xi \xi}}{\psi}-\frac{\psi_{\xi}^{2}}{\psi^{2}}\right) .
\end{aligned}
$$

Then (29) can be written as

$$
\begin{aligned}
& A_{1}^{2}\left(\frac{\psi_{\xi \xi}}{\psi}-\frac{\psi_{\xi}^{2}}{\psi^{2}}\right)^{2}+4 c_{2}\left(A_{0}+A_{1} \frac{\psi_{\xi}}{\psi}\right)^{2} \\
& \quad+4 c_{4}\left(A_{0}+A_{1} \frac{\psi_{\xi}}{\psi}\right)^{3}+4 c_{6}\left(A_{0}+A_{1} \frac{\psi_{\xi}}{\psi}\right)^{4} \\
& =0 .
\end{aligned}
$$

Then (41) can be written as

$$
\begin{aligned}
& A_{1}^{2}\left[\frac{\psi_{\xi \xi}^{2}}{\psi^{2}}-2 \psi_{\xi \xi} \frac{\psi_{\xi}^{2}}{\psi^{3}}+\frac{\psi_{\xi}^{4}}{\psi^{4}}\right]+4 c_{2}\left[A_{0}^{2}\right. \\
& \left.+2 A_{0} A_{1} \frac{\psi_{\xi}}{\psi}+A_{1}{ }^{2} \frac{\psi_{\xi}{ }^{2}}{\psi^{2}}\right]+4 c_{4}\left[A_{0}{ }^{3}\right. \\
& \left.+3 A_{0}{ }^{2} A_{1} \frac{\psi_{\xi}}{\psi}+3 A_{0} A_{1}{ }^{2} \frac{\psi_{\xi}^{2}}{\psi^{2}}+A_{1}{ }^{3} \frac{\psi_{\xi}}{\psi^{3}}\right] \\
& +4 c_{6}\left[A_{0}{ }^{4}+4 A_{0}{ }^{3} A_{1} \frac{\psi_{\xi}}{\psi}+6 A_{0}{ }^{2} A_{1}{ }^{2} \frac{\psi_{\xi}^{2}}{\psi^{2}}\right. \\
& \left.+4 A_{0} A_{1}{ }^{3} \frac{\psi_{\xi}{ }^{3}}{\psi^{3}}+A_{1}{ }^{4} \frac{\psi_{\xi}}{\psi^{4}}\right]=0 .
\end{aligned}
$$

Equating expressions in (42) at $\psi^{-1}, \psi^{-2}, \psi^{-3}$, and $\psi^{-4}$ to zero, we have the following system of equations:

$$
\begin{array}{r}
{\left[1+4 c_{6} A_{1}^{2}\right] A_{1}^{2}=0} \\
{\left[\psi_{\xi \xi}-2 A_{1}\left\{c_{4}+4 c_{6} A_{0}\right\} \psi_{\xi}\right] A_{1}^{2}=0} \\
{\left[\psi_{\xi \xi}{ }^{2}+4\left\{c_{2}+3 c_{4} A_{0}+6 c_{6} A_{0}{ }^{2}\right\} \psi_{\xi}^{2}\right] A_{1}{ }^{2}=0} \\
\left\{2 c_{2}+3 c_{4} A_{0}+4 c_{6} A_{0}{ }^{2}\right\} A_{0} A_{1}=0
\end{array}
$$

Solving the system of equations in (43),

$$
A_{1}=\mp i \frac{k}{2} \sqrt{\frac{3 \alpha}{\gamma}}
$$

Family 1

$$
\begin{aligned}
& A_{0}=\frac{-\beta+\sqrt{\beta^{2}-16 \gamma \beta\left[\alpha k_{1}{ }^{3}-k_{1}{ }^{2}-k_{2}\right] / 3}}{4 \gamma / 3}, \\
& \psi_{1}(\xi)=\varepsilon_{11}+\varepsilon_{12} e^{2 i \sqrt{c_{2}+3 c_{4} A_{0}+6 c_{6} A_{0}{ }^{2}} \xi}, \\
& q_{1}(x, t)=e^{i\left[k_{1} x+k_{2} t\right]}\left\{A_{0}\right.
\end{aligned}
$$

$$
\begin{aligned}
& -k \varepsilon_{12} \sqrt{\frac{3 \alpha\left(c_{2}+3 c_{4} A_{0}+6 c_{6} A_{0}^{2}\right)}{\gamma}} \\
& \left.\cdot \frac{e^{2 i \sqrt{c_{2}+3 c_{4} A_{0}+6 c_{6} A_{0}^{2}}(k x-\lambda t)}}{\varepsilon_{11}+\varepsilon_{12} e^{2 i \sqrt{c_{2}+3 c_{4} A_{0}+6 c_{6} A_{0}^{2}}(k x-\lambda t)}}\right\}^{1 / 2} .
\end{aligned}
$$


Family 2

$$
\begin{aligned}
& A_{0}=\frac{-\beta-\sqrt{\beta^{2}-16 \gamma \beta\left[\alpha k_{1}^{3}-k_{1}^{2}-k_{2}\right] / 3}}{4 \gamma / 3}, \\
& \psi_{2}(\xi)=\varepsilon_{21}+\varepsilon_{22} e^{2 i \sqrt{c_{2}+3 c_{4} A_{0}+6 c_{6} A_{0}^{2}} \xi}, \\
& q_{2}(x, t)=e^{i\left[k_{1} x+k_{2} t\right]}\left\{A_{0}\right. \\
& -k \varepsilon_{22} \sqrt{\frac{3 \alpha\left(c_{2}+3 c_{4} A_{0}+6 c_{6} A_{0}^{2}\right)}{\gamma}} \\
& \left.\cdot \frac{e^{2 i \sqrt{c_{2}+3 c_{4} A_{0}+6 c_{6} A_{0}^{2}}(k x-\lambda t)}}{\varepsilon_{21}+\varepsilon_{22} e^{2 i \sqrt{c_{2}+3 c_{4} A_{0}+6 c_{6} A_{0}^{2}}(k x-\lambda t)}}\right\}^{1 / 2} .
\end{aligned}
$$

Family 3

$$
\begin{aligned}
& A_{0}=\frac{-3 \beta+\sqrt{9 \beta^{2}-128 \beta \gamma\left[\alpha k_{1}^{3}-k_{1}^{2}-k_{2}\right] / 3}}{16 \gamma / 3} \\
& \psi_{3}(\xi)=\varepsilon_{31}+\varepsilon_{32} e^{2 i \sqrt{c_{2}+3 c_{4} A_{0}+6 c_{6} A_{0}^{2}} \xi} \\
& q_{3}(x, t)=e^{i\left[k_{1} x+k_{2} t\right]}\left\{A_{0}\right. \\
& -k \varepsilon_{32} \sqrt{\frac{3 \alpha\left(c_{2}+3 c_{4} A_{0}+6 c_{6} A_{0}^{2}\right)}{\gamma}} \\
& \left.\cdot \frac{e^{2 i \sqrt{c_{2}+3 c_{4} A_{0}+6 c_{6} A_{0}^{2}}(k x-\lambda t)}}{\varepsilon_{31}+\varepsilon_{32} e^{2 i \sqrt{c_{2}+3 c_{4} A_{0}+6 c_{6} A_{0}^{2}}(k x-\lambda t)}}\right\}^{1 / 2}
\end{aligned}
$$

Family 4

$$
\begin{aligned}
& A_{0}=\frac{-3 \beta-\sqrt{9 \beta^{2}-128 \beta \gamma\left[\alpha k_{1}^{3}-k_{1}^{2}-k_{2}\right] / 3}}{16 \gamma / 3} \\
& \psi_{4}(\xi)=\varepsilon_{41}+\varepsilon_{42} e^{2 i \sqrt{c_{2}+3 c_{4} A_{0}+6 c_{6} A_{0}^{2}} \xi} \\
& q_{4}(x, t)=e^{i\left[k_{1} x+k_{2} t\right]}\left\{A_{0}\right. \\
& -k \varepsilon_{42} \sqrt{\frac{3 \alpha\left(c_{2}+3 c_{4} A_{0}+6 c_{6} A_{0}^{2}\right)}{\gamma}} \\
& \left.\cdot \frac{e^{2 i \sqrt{c_{2}+3 c_{4} A_{0}+6 c_{6} A_{0}^{2}}(k x-\lambda t)}}{\varepsilon_{41}+\varepsilon_{42} e^{2 i \sqrt{c_{2}+3 c_{4} A_{0}+6 c_{6} A_{0}^{2}}(k x-\lambda t)}}\right\}^{1 / 2}
\end{aligned}
$$

Family 5

$$
\begin{aligned}
& A_{0}=\frac{-\beta+\sqrt{\beta^{2}-16 \gamma \beta\left[\alpha k_{1}^{3}-k_{1}^{2}-k_{2}\right] / 3}}{4 \gamma / 3}, \\
& \psi_{5}(\xi)=\varepsilon_{51}+\varepsilon_{52} e^{-2 i \sqrt{c_{2}+3 c_{4} A_{0}+6 c_{6} A_{0}{ }^{2}} \xi}, \\
& q_{5}(x, t)=e^{i\left[k_{1} x+k_{2} t\right]}\left\{A_{0}\right. \\
& +k \varepsilon_{52} \sqrt{\frac{3 \alpha\left(c_{2}+3 c_{4} A_{0}+6 c_{6} A_{0}^{2}\right)}{\gamma}} \\
& \left.\cdot \frac{e^{-2 i \sqrt{c_{2}+3 c_{4} A_{0}+6 c_{6} A_{0}{ }^{2}}(k x-\lambda t)}}{\varepsilon_{51}+\varepsilon_{52} e^{-2 i \sqrt{c_{2}+3 c_{4} A_{0}+6 c_{6} A_{0}^{2}}(k x-\lambda t)}}\right\}^{1 / 2} .
\end{aligned}
$$

Family 6

$$
\begin{aligned}
& A_{0}=\frac{-\beta-\sqrt{\beta^{2}-16 \gamma \beta\left[\alpha k_{1}^{3}-k_{1}^{2}-k_{2}\right] / 3}}{4 \gamma / 3}, \\
& \psi_{6}(\xi)=\varepsilon_{61}+\varepsilon_{62} e^{-2 i \sqrt{c_{2}+3 c_{4} A_{0}+6 c_{6} A_{0}{ }^{2}} \xi}, \\
& q_{6}(x, t)=e^{i\left[k_{1} x+k_{2} t\right]}\left\{A_{0}\right. \\
& +k \varepsilon_{62} \sqrt{\frac{3 \alpha\left(c_{2}+3 c_{4} A_{0}+6 c_{6} A_{0}^{2}\right)}{\gamma}} \\
& \left.\cdot \frac{e^{-2 i \sqrt{c_{2}+3 c_{4} A_{0}+6 c_{6} A_{0}{ }^{2}}(k x-\lambda t)}}{\varepsilon_{61}+\varepsilon_{62} e^{-2 i \sqrt{c_{2}+3 c_{4} A_{0}+6 c_{6} A_{0}{ }^{2}}(k x-\lambda t)}}\right\}^{1 / 2} .
\end{aligned}
$$

Family 7

$$
\begin{aligned}
& A_{0}=\frac{-3 \beta+\sqrt{9 \beta^{2}-128 \beta \gamma\left[\alpha k_{1}^{3}-k_{1}^{2}-k_{2}\right] / 3}}{16 \gamma / 3}, \\
& \psi_{7}(\xi)=\varepsilon_{71}+\varepsilon_{72} e^{2 i \sqrt{c_{2}+3 c_{4} A_{0}+6 c_{6} A_{0}^{2}} \xi}, \\
& q_{7}(x, t)=e^{i\left[k_{1} x+k_{2} t\right]}\left\{A_{0}\right. \\
& +k \varepsilon_{72} \sqrt{\frac{3 \alpha\left(c_{2}+3 c_{4} A_{0}+6 c_{6} A_{0}^{2}\right)}{\gamma}} \\
& \left.\cdot \frac{e^{-2 i \sqrt{c_{2}+3 c_{4} A_{0}+6 c_{6} A_{0}^{2}}(k x-\lambda t)}}{\varepsilon_{71}+\varepsilon_{72} e^{-2 i \sqrt{c_{2}+3 c_{4} A_{0}+6 c_{6} A_{0}^{2}}(k x-\lambda t)}}\right\}^{1 / 2} .
\end{aligned}
$$


Family 8

$$
\begin{aligned}
& A_{0}=\frac{-3 \beta-\sqrt{9 \beta^{2}-128 \beta \gamma\left[\alpha k_{1}^{3}-k_{1}^{2}-k_{2}\right] / 3}}{16 \gamma / 3} \\
& \psi_{8}(\xi)=\varepsilon_{81}+\varepsilon_{82} e^{2 i \sqrt{c_{2}+3 c_{4} A_{0}+6 c_{6} A_{0}^{2}} \xi} \\
& q_{8}(x, t)=e^{i\left[k_{1} x+k_{2} t\right]}\left\{A_{0}\right. \\
& +k \varepsilon_{82} \sqrt{\frac{3 \alpha\left(c_{2}+3 c_{4} A_{0}+6 c_{6} A_{0}^{2}\right)}{\gamma}} \\
& \left.\cdot \frac{e^{-2 i \sqrt{c_{2}+3 c_{4} A_{0}+6 c_{6} A_{0}^{2}}(k x-\lambda t)}}{\varepsilon_{81}+\varepsilon_{82} e^{-2 i \sqrt{c_{2}+3 c_{4} A_{0}+6 c_{6} A_{0}^{2}}(k x-\lambda t)}}\right\}^{1 / 2} .
\end{aligned}
$$

Family 9

$$
\begin{aligned}
& A_{0}=\frac{-\beta+\sqrt{\beta^{2}-16 \gamma \beta\left[\alpha k_{1}^{3}-k_{1}^{2}-k_{2}\right] / 3}}{4 \gamma / 3}, \\
& \psi_{9}(\xi)=\varepsilon_{91}+\varepsilon_{92} e^{i k \sqrt{3 \alpha / \gamma}\left[c_{4}+2 c_{6} A_{0}\right] \xi}, \\
& q_{9}(x, t)=e^{i\left[k_{1} x+k_{2} t\right]}\left\{A_{0}-\frac{3 k^{2} \alpha}{\gamma}\left[c_{4}+2 c_{6} A_{0}\right]^{2}\right. \\
& \left.\cdot \frac{\varepsilon_{92} e^{i k \sqrt{3 \alpha / \gamma}\left[c_{4}+2 c_{6} A_{0}\right](k x-\lambda t)}}{\varepsilon_{91}+\varepsilon_{92} e^{i k \sqrt{3 \alpha / \gamma}\left[c_{4}+2 c_{6} A_{0}\right](k x-\lambda t)}}\right\}^{1 / 2} .
\end{aligned}
$$

Family 10

$$
\begin{aligned}
& A_{0}=\frac{-\beta-\sqrt{\beta^{2}-16 \gamma \beta\left[\alpha k_{1}^{3}-k_{1}^{2}-k_{2}\right] / 3}}{4 \gamma / 3}, \\
& \psi_{10}(\xi)=\varepsilon_{101}+\varepsilon_{102} e^{i k \sqrt{3 \alpha / \gamma}\left[c_{4}+2 c_{6} A_{0}\right] \xi}, \\
& q_{10}(x, t)=e^{i\left[k_{1} x+k_{2} t\right]}\left\{A_{0}-\frac{3 k^{2} \alpha}{\gamma}\left[c_{4}+2 c_{6} A_{0}\right]^{2}\right. \\
& \left.\cdot \frac{\varepsilon_{102} e^{i k \sqrt{3 \alpha / \gamma}\left[c_{4}+2 c_{6} A_{0}\right](k x-\lambda t)}}{\varepsilon_{101}+\varepsilon_{102} e^{i k \sqrt{3 \alpha / \gamma}\left[c_{4}+2 c_{6} A_{0}\right](k x-\lambda t)}}\right\}^{1 / 2} .
\end{aligned}
$$

Family 11

$$
\begin{aligned}
& A_{0}=\frac{-3 \beta+\sqrt{9 \beta^{2}-128 \beta \gamma\left[\alpha k_{1}^{3}-k_{1}{ }^{2}-k_{2}\right] / 3}}{16 \gamma / 3}, \\
& \psi_{11}(\xi)=\varepsilon_{111}+\varepsilon_{112} e^{i k \sqrt{3 \alpha / \gamma}\left[c_{4}+2 c_{6} A_{0}\right] \xi}, \\
& q_{11}(x, t)=e^{i\left[k_{1} x+k_{2} t\right]}\left\{A_{0}-\frac{3 k^{2} \alpha}{\gamma}\left[c_{4}+2 c_{6} A_{0}\right]^{2}\right. \\
& \left.\cdot \frac{\varepsilon_{112} e^{i k \sqrt{3 \alpha / \gamma}\left[c_{4}+2 c_{6} A_{0}\right](k x-\lambda t)}}{\varepsilon_{111}+\varepsilon_{112} e^{i k \sqrt{3 \alpha / \gamma}\left[c_{4}+2 c_{6} A_{0}\right](k x-\lambda t)}}\right\}^{1 / 2} .
\end{aligned}
$$

Family 12

$$
\begin{aligned}
& A_{0}=\frac{-3 \beta-\sqrt{9 \beta^{2}-128 \beta \gamma\left[\alpha k_{1}^{3}-k_{1}^{2}-k_{2}\right] / 3}}{16 \gamma / 3}, \\
& \psi_{12}(\xi)=\varepsilon_{121}+\varepsilon_{122} e^{i k \sqrt{3 \alpha / \gamma}\left[c_{4}+2 c_{6} A_{0}\right] \xi}, \\
& q_{12}(x, t)=e^{i\left[k_{1} x+k_{2} t\right]}\left\{A_{0}-\frac{3 k^{2} \alpha}{\gamma}\left[c_{4}+2 c_{6} A_{0}\right]^{2}\right. \\
& \left.\cdot \frac{\varepsilon_{122} e^{i k \sqrt{3 \alpha / \gamma}\left[c_{4}+2 c_{6} A_{0}\right](k x-\lambda t)}}{\varepsilon_{121}+\varepsilon_{122} e^{i k \sqrt{3 \alpha / \gamma}\left[c_{4}+2 c_{6} A_{0}\right](k x-\lambda t)}}\right\}^{1 / 2} .
\end{aligned}
$$

\section{Conclusion}

In this paper, series of new traveling wave solutions have been obtained. The Csch method and the Extended Tanh-Coth method and modified simple equation method are used to carry out the integration of the generalized NLS equation, which is RKL. These methods can be also applied to solve other types of the generalized nonlinear evolution equations with complex coefficients. The solitary waves in Figures 1 and 2 obtained by the Csch and Tanh-Coth methods, respectively, are identical in form and behavior. The obtained solutions are very useful and may be important to explain some physical phenomena and find applications in the nonlinear pulse propagation through optical fibers.

\section{Conflicts of Interest}

The author declares that there are no conflicts of interest regarding the publication of this paper.

\section{Acknowledgments}

This research has been supported by Al-Rafidain University College. 


\section{References}

[1] R. Radhakrishnan, A. Kundu, and M. Lakshmanan, "Coupled nonlinear Schrödinger equations with cubic-quintic nonlinearity: integrability and soliton interaction in non-Kerr media," Physical Review E: Statistical, Nonlinear, and Soft Matter Physics, vol. 60, no. 3, pp. 3314-3323, 1999.

[2] W.-P. Hong, "Optical solitary wave solutions for the higher order nonlinear Schrödinger equation with cubic-quintic nonKerr terms," Optics Communications, vol. 194, no. 1-3, pp. 217223, 2001.

[3] M. Wang, X. Li, and J. Zhang, "Sub-ODE method and solitary wave solutions for higher order nonlinear Schrödinger equation," Physics Letters A, vol. 363, no. 1-2, pp. 96-101, 2007.

[4] H. Triki and T. R. Taha, "Exact analytic solitary wave solutions for the RKL model," Mathematics and Computers in Simulation, vol. 80, no. 4, pp. 849-854, 2009.

[5] W. van Saarloos and P. C. Hohenberg, "Fronts, pulses, sources and sinks in generalized complex Ginzburg-Landau equations," Physica D: Nonlinear Phenomena, vol. 56, no. 4, pp. 303-367, 1992.

[6] A. Kundu, "Landau-Lifshitz and higher-order nonlinear systems gauge generated from nonlinear Schrödinger type equations," Journal of Mathematical Physics, vol. 25, no. 12, pp. 34333438, 1984.

[7] A. Kundu, "Exact solutions to higher-order nonlinear equations through gauge transformation," Physica D: Nonlinear Phenomena, vol. 25, no. 1-3, pp. 399-406, 1987.

[8] G. Ebadi and A. Biswas, "The $\left(G^{\prime} / G\right)$ method and 1-soliton solution of the Davey-Stewartson equation," Mathematical and Computer Modelling, vol. 53, no. 5-6, pp. 694-698, 2011.

[9] G. Ebadia and E. V. Krishnanb, "Manel Labidic, Essaid Zerradd and Anjan Biswas," Analytical and numerical solutions to the Davey-Stewartson equation with power-law nonlinearity, Waves in Random and Complex Media, vol. 21, no. 4, pp. 559-590, November 2011.

[10] G.-q. Xu, "Extended auxiliary equation method and its applications to three generalized NLS equations," Abstract and Applied Analysis, vol. 2014, Article ID 541370, 7 pages, 2014.

[11] A. J. M. Jawad, M. Mirzazadeh, Q. Zhou, and A. Biswas, "Optical solitons with anti-cubic nonlinearity using three integration schemes," Superlattices and Microstructures, vol. 105, pp. 1-10, 2017.

[12] A. J. M. Jawad, M. D. Petkovic, and A. Biswas, "Soliton solutions to a few coupled nonlinear wave equations by tanh method," Iranian Journal of Science \& Technology, vol. 37, no. 2, pp. 109115, 2013.

[13] A. J. M. Jawad, "Tan Method for Solitary Wave Solutions for Nonlinear Evolution Equations," in Proceedings of the International Arab conference of Mathematics in Jordon, 2014.

[14] A. J. M. Jawad, M. D. Petković, and A. Biswas, "Modified simple equation method for nonlinear evolution equations," Applied Mathematics and Computation, vol. 217, no. 1, pp. 869-877, 2010. 


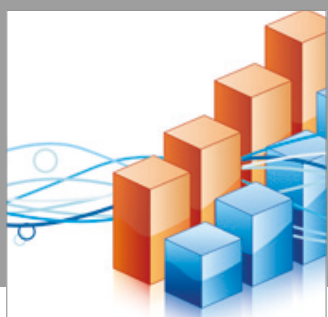

Advances in

Operations Research

vatersals

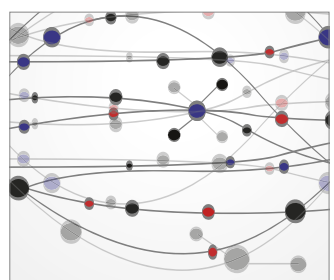

\section{The Scientific} World Journal
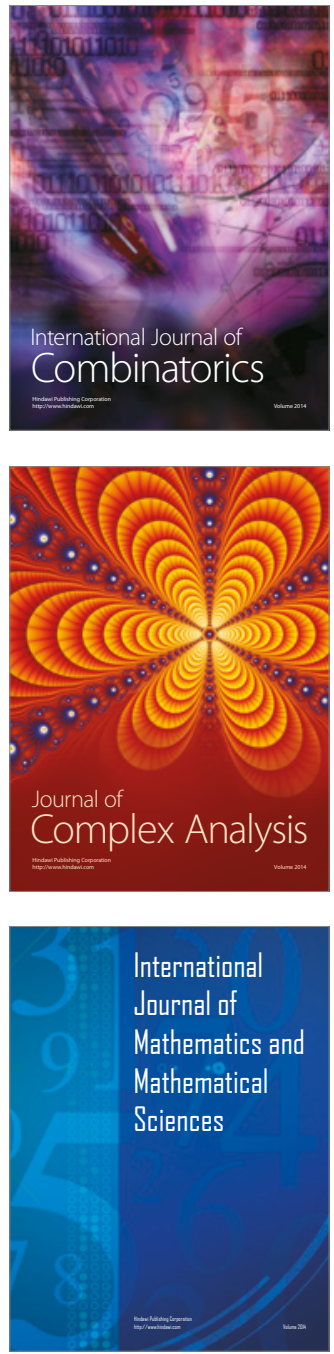
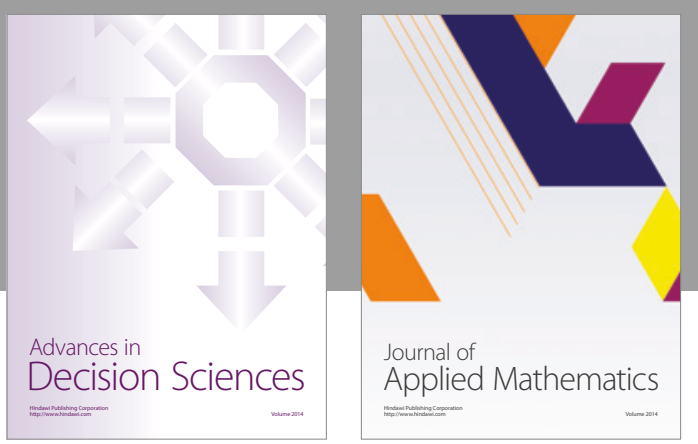

Algebra

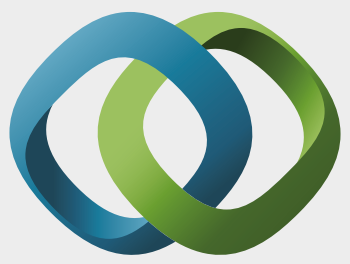

\section{Hindawi}

Submit your manuscripts at

https://www.hindawi.com
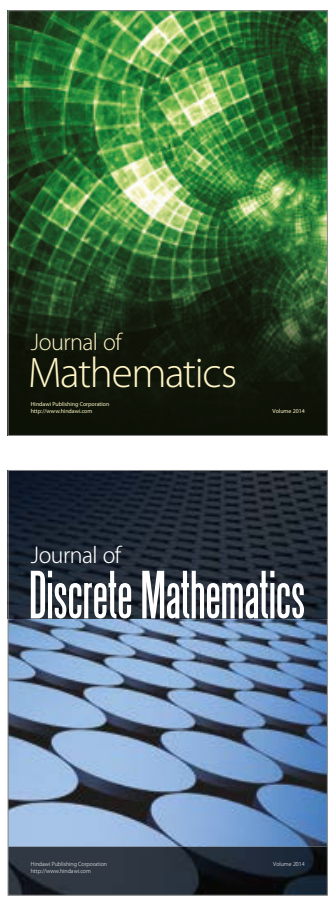

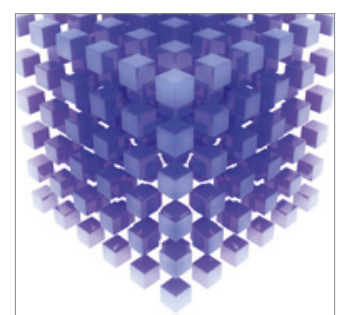

Mathematical Problems in Engineering
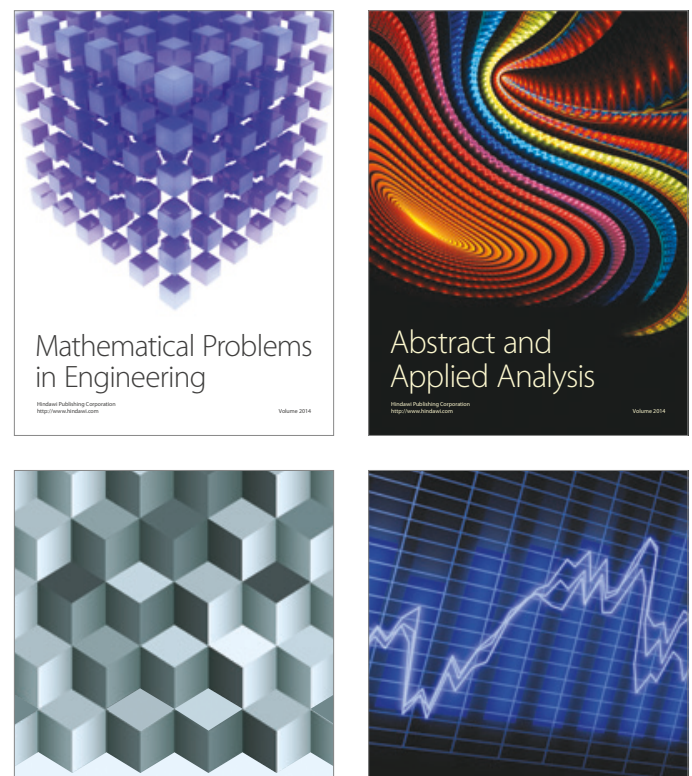

Journal of

Function Spaces

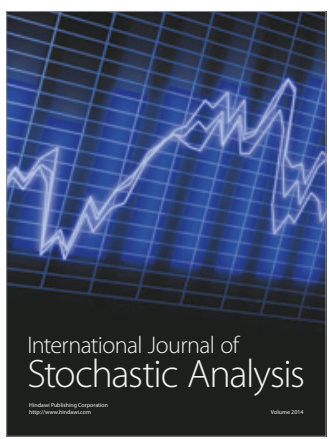

Probability and Statistics
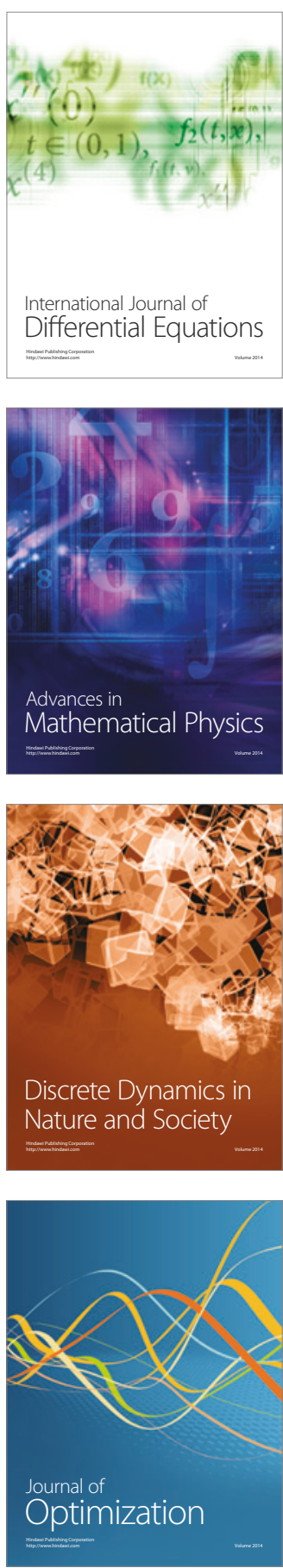